\title{
Novel Small Molecule Inhibitors of Cancer Stem Cell Signaling Pathways
}

Danysh Abetov, Zhanar Mustapova, Timur Saliev, Denis Bulanin, Kanat Batyrbekov, Charles P. Gilman

Laboratory of translational medicine and Life Sciences Technologies, Department of Biomedical Sciences

\section{Abstract}

The main aim of oncologists worldwide is to understand and then intervene in the primary tumor initiation and propagation mechanisms. This is essential to allow targeted elimination of cancer cells without altering normal mitotic cells. Currently, there are two main rival theories describing the process of tumorigenesis. According to the Stochastic Model, potentially any cell, once defunct, is capable of initiating carcinogenesis. Alternatively the Cancer Stem Cell (CSC) Model posits that only a small fraction of undifferentiated tumor cells are capable of triggering carcinogenesis. Like healthy stem cells, CSCs are also characterized by a capacity for self-renewal and the ability to generate differentiated progeny, possibly mediating treatment resistance, thus leading to tumor recurrence and metastasis. Moreover, molecular signaling profiles are similar between CSCs and normal stem cells, including Wnt, Notch and Hedgehog pathways. Therefore, development of novel chemotherapeutic agents and proteins (e.g., enzymes and antibodies) specifically targeting CSCs are attractive pharmaceutical candidates. This article describes small molecule inhibitors of stem cell pathways Wnt, Notch and Hedgehog, and their recent chemotherapy clinical trials.

Original language English

Pages (from-to) 9 909-918

Number of pages $\quad 10$

Journal $\quad \underline{\text { Stem Cell Reviews and Reports }}$

Volume $\quad 11$

Issue number 6

State Published - Dec 12015

Abetov, D., Mustapova, Z., Saliev, T., Bulanin, D., Batyrbekov, K., \& Gilman, C. P. (2015). Novel Small Molecule Inhibitors of Cancer Stem Cell Signaling Pathways. Stem Cell Reviews and Reports, 11(6), 909-918. DOI: 10.1007/s12015-015-9612-X 